\title{
Physiology of the Basal Ganglia and Pathophysiology of Parkinson's Disease
}

\author{
Robert G. Lee
}

\begin{abstract}
This paper reviews current concepts concerning the anatomical organization and functions of the basal ganglia focusing mainly on the role of the basal ganglia in control of movement. Results of several studies which have been carried out on patients with Parkinson's disease are also reviewed and discussed as examples of altered normal physiological mechanisms.
\end{abstract}

RÉSUMÉ: Pathologie des noyaux gris centraux et pathophysiologie de la maladie de Parkinson. Cet article revoit les concepts actuels sur l'organisation et les fonctions des noyaux gris centraux, en accordant une attention particulière au rôle des noyaux gris centraux dans le contrôle du mouvement. Nous revoyons également les résultats de plusieurs études qui ont été effectuées sur des patients atteints de la maladie de Parkinson et nous en discutons en tant qu'exemples d'altérations des mécanismes physiologiques normaux.

Can. J. Neurol. Sci. 1987; 14:373-380

Long before much was known about the anatomy and physiology of the basal ganglia, it was recognized that pathology in this part of the brain often caused disorders of muscle tone and movement. However, exactly what role the basal ganglia played in normal movement remained a mystery. In recent years, the results of studies using a combination of anatomical, physiological, and neurochemical techniques have begun to shed new light on this complex area of the central nervous system. With a better understanding of normal function it is now possible to begin a more systematic analysis of the various disorders of movement associated with disease of the basal ganglia.

Several recent reviews and monographs have discussed the functions of the basal ganglia. ${ }^{1-5}$ Other contributions to this symposium will deal with the chemical and pharmacological aspects of basal ganglia functions. This paper will provide a selective review of some of the recent anatomical and physiological developments and will attempt to relate these to some of the abnormalities observed in patients with Parkinson's disease.

\section{ANATOMICAL OVERVIEW}

The general anatomical organisation of the basal ganglia is now fairly well understood. The striatum, which is the major input centre for the basal ganglia, receives projections from almost all areas of the cerebral cortex (Figure 1). After some internal signal processing outputs are directed from the internal segment of globus pallidus (GPi) and pars reticulata of substantia nigra (SNpr) through the thalamus to more restricted zones of cerebral cortex, mainly in the frontal lobe.
The arrangement illustrated in Figure 1 emphasizes the concept which has been proposed by several investigators, namely that the basal ganglia act like a funnel collecting information from widely scattered areas of cortex and gradually consolidating this into a more focused output directed to a specific area. In addition to the inputs from cerebral cortex, the striatum receives afferents from a number of sites in the brain stem and diencephalon including centrum medianum, pars compacta of the substantia nigra $(\mathrm{SNpc})$ and the raphe nuclei. These inputs are presumably able to modulate the flow of information arriving via the corticostriate projections. There is no doubt that the basal ganglia play an important role in motor function, but it is becoming increasingly obvious that control of movement is not the only function of the basal ganglia. Some areas such as the caudate have no obvious motor role and many of the projections from the basal ganglia are directed back to cortical areas which do not seem to be involved in control of movement. There is increasing evidence that some parts of the basal ganglia play a role in cognitive function (see following paper by Phillips). The anatomical link between the ventral striatum and parts of the limbic system suggests that the basal ganglia may be involved with functions such as mood and motivation.

It has been proposed that, in addition to the motor circuit which projects back to motor areas of cortex, the basal ganglia also include a complex circuit receiving input from association areas of cortex and projecting back to prefrontal areas. One concept which has emerged is that the basal ganglia may provide a mechanism whereby cortical regions involved in "higher" brain functions may gain access to motor output areas. ${ }^{6}$ 


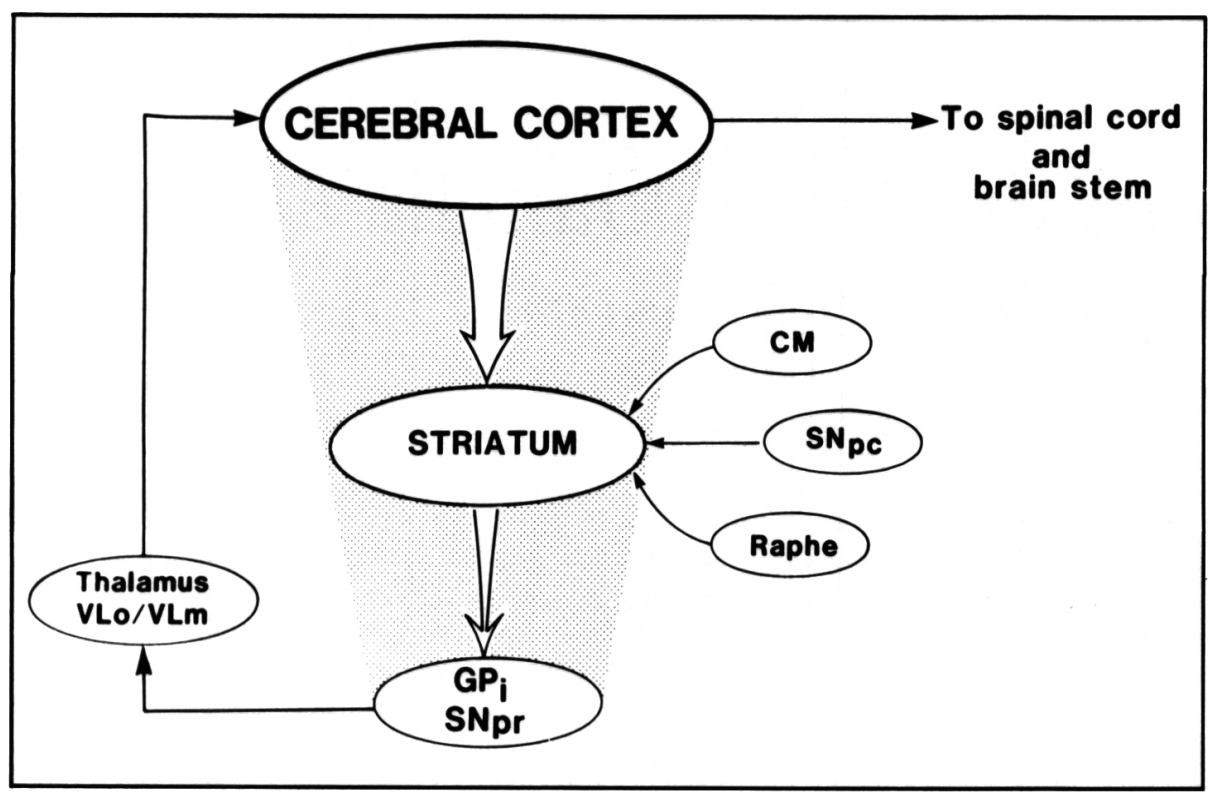

Figure 1 - Simplified diagram showing the general organization of the basal ganglia. Afferent input from the cerebral cortex and several brain stem nuclei is directed to the striatum. The major outflow from the basal ganglia originatesfrom globuspallidus and substantia nigra (pr) and is directed back to cerebral cortex.

The concept of multiple functions for the basal ganglia has recently been further developed by Alexander, et al. ${ }^{7}$ who describe at least five parallel but functionally segregated subcortical loops passing through the basal ganglia. These circuits are identified by the cortical region to which the outputs are directed and include: (1) The motor circuit projecting to the supplementary motor area (SMA), (2) an oculomotor circuit projecting to area 8 , (3) an anterior cingulate circuit receiving input from hippocampus, amygdala, and other limbic areas and sending projections back to the anterior portion of the cingulate gyrus. In addition, there is anatomical evidence for two additional separate circuits projecting through the basal ganglia to prefrontal cortex, one to the dorsolateral prefrontal area and the other to the lateral orbitofrontal region. Our knowledge of the function of these non-motor circuits is still quite limited, and the remainder of this discussion will focus on the motor functions of the basal ganglia.

The inputs for the motor circuit come mainly from sensorimotor cortex, premotor cortex, and some areas of the parietal lobe and are directed to the putamen. The output from globus pallidus goes to the thalamus (VLm/VLo) and from there almost exclusively back to SMA. It is interesting to note that some functions such as motor planning and programming which have tentatively been assigned to SMA are similar to functions which others have proposed for the basal ganglia. Furthermore, lesions of SMA can produce motor deficits which are similar in several respects to what is observed in Parkinson's disease. ${ }^{6}$

It is useful to compare and contrast the basal ganglia with the cerebellum, the other major subcortical system concerned with control of movement. The basal ganglia receive no direct sensory input from the periphery, whereas the cerebellum receives extensive projections via the dorsal columns and spinocerebellar tracts from muscles, joints, and cutaneous receptors. As already indicated the major projection from the motor components of the basal ganglia goes to SMA; much of the cortically directed output from cerebellum goes directly to motor cortex. The basal ganglia influence motor output almost entirely through their effect on cerebral cortex; there are no direct projections from the basal ganglia extending below the upper brainstem, and those that do reach the brainstem seem to be concerned mainly with eye movements rather than with posture or limb movements. On the other hand there are extensive connections between the cerebellum and brainstem nuclei which give rise to descending motor pathways such as the reticulospinal and vestibulospinal tracts. Much of the output from the basal ganglia and cerebellum passes through the VL/VA region of the thalamus, and at one time it was thought that there might be convergence of input from cerebellum and basal ganglia on to single thalamic neurons. However, anatomical studies now indicate that the thalamic targets for the basal ganglia and cerebellar outputs are largely separate. ${ }^{8}$

\section{INTERNAL STRUCTURE}

There is a tendency amongst clinicians, and even some physiologists, to view the striatum as a black box without much concern about what goes on inside. The reluctance to open this black box can be partially appreciated when we consider the complexity of the internal structure and neuronal connections within the striatum. The details of the internal structure of the striatum are beyond the scope of this review, but we shall consider briefly a few main points. Golgi impregnation studies have revealed at least nine morphologically distinct neurons within the striatum. More than a dozen putative neurotransmittors have been identified and there are nine distinct types of synapses. ${ }^{9}$

Some of the major cell types in the neostriatum are listed in Table 1. These can be classified according to their size and the presence or absence of dendritic spines in Golgi preparations, and also by immunocytochemical studies to identify their neurotransmitters. The most commonly encountered cell in the neostriatum is the medium size densely spiny neuron. This is probably the major output cell of the striatum, sending projections to globus pallidus and substantia nigra as well as collaterals to other striatal neurons. These cells utilize GABA, substance $P$, and enkephalin as neurotransmitters. There is some evidence that GABA and enkephalin may co-exist in the same cell. A second cell type is much larger and has no dendritic spines. This cell is relatively rare in Golgi preparations and is found only in the ventral striatum. It is a cholinergic cell and sends projection to the substantia nigra. There are also several types 
of interneurons which utilize acetylcholine, GABA, and somatostatin as transmitters.

The inputs from the cerebral cortex which are excitatory and use glutamate as a transmitter probably terminate on all cell types in the striatum although the medium size densely spiny output neurons and the cholinergic interneurons are important targets. It is likely that the dopaminergic inputs from SNpc form synapses on the same neurons and there is evidence that nigrostriatal terminals produce both post-synaptic inhibition of striatal neurons and pre-synaptic inhibition of corticostriatal nerve terminals. ${ }^{10}$ However, we are still a long way from undertanding how these neuronal interactions function in normal movement or how loss of the nigrostriatal dopaminergic influences results in the motor deficits seen in Parkinson's disease.

\section{Microphysiology of THE Basal Ganglia}

Initial approaches to understand the function of the basal ganglia using the classical techniques of electrical stimulation and lesion making produced results which were disappointing and difficult to interpret (see DeLong and Georgopoulos for detailed review). 'Even single cell recording in behaving primates, a technique which has contributed a great deal to our knowledge of cortical motor areas and the cerebellum, has been slow to yield information concerning the function of basal ganglia. There are at least two reasons for this. First, there is not yet any reliable method for identifying which of the many cell types in the basal ganglia is being recorded from when a microelectrode is inserted. Even after recording from large numbers of cells it is difficult to develop a comprehensive picture of single cell function when the population is a heterogeneous group including output neurons and various types of interneurons. Secondly, neurons in most areas of the basal ganglia show a much less precise relationship to specific body movements than neurons in motor cortex, cerebellum, and other motor centres. Despite these difficulties a number of investigators, particularly Mahlon DeLong and his colleagues at Johns Hopkins, have carried out extensive single cell recordings from various sites in the basal ganglia and, although the puzzle is far from complete, the parts are beginning to fit together in a manner which allows at least a glimpse of how the total picture may eventually appear.

\section{Spontaneous activity of single neurons}

The discharge properties of single neurons in different regions of the basal ganglia are summarized in Table 2. Most of the studies which have been done have focused on either the input centre, the striatum, or the output areas in GPi and SNpr. There is a striking difference in the spontaneous activity of neurons in these two areas. In the GPi and SNpr there is a high level of spontaneous activity with sustained high frequency discharges. " In GPe, there is also high frequency spontaneous activity but more of a tendency for cells to fire in bursts. In the putamen, by contrast, many cells are either silent at rest or have low spontaneous firing rates. ${ }^{12}$

\section{Relationship between neuronal discharges and limb movements}

In both the putamen and globus pallidus about $50 \%$ of neurons which have been studied show some type of relationship to limb movements. The changes in neuronal discharges which accompany movements show the best correlation with direc- tion of movement. ${ }^{13}$ Figure 2 shows an example of a single neuron in the putamen of a monkey performing flexion and extension movements of the elbow. There is a marked increase in discharge frequency preceding and following the onset of elbow extension, but no changes occur with elbow flexion. The increased firing is slightly more pronounced when there is a larger load opposing the movement, but the correlation between neuronal activity in the putamen and the force generated by the movement is much less than in other centres such as the motor cortex. These observations suggest that some neurons in the putamen are involved primarily in specifying the direction and scaling the amplitude of voluntary movements.

Although many neurons in the basal ganglia do show movement associated changes in discharge frequency, the timing of these changes is much less tightly coupled to movement onset than is the case for neurons in the motor cortex or cerebellum. Figure 3 shows the timing of neuronal discharges in relation to movement onset for a large number of neurons in the putamen. Although some of these cells increase firing frequency in advance of the movement many do not show any change until after the movement has actually commenced. By contrast, the majority of neurons in cerebellum and motor cortex which are associated with movements show an increase in firing prior to movement onset.

With this imprecise relationship to movement onset, it seems

Table 1: Different Types of Neurons Identified in the Neostriatum

\begin{tabular}{lll}
\hline \hline OUTPUT NEURONS & Projection sites & Neurotransmitters \\
\hline $\begin{array}{l}\text { Medium size densely } \\
\text { spiny neurons }\end{array}$ & $\begin{array}{l}\text { Globus pallidus } \\
\text { Substantia nigra } \\
\text { Other striatal } \\
\text { neurons } \\
\text { Substantia nigra }\end{array}$ & $\begin{array}{l}\text { GABA } \\
\text { Substance P } \\
\text { Enkephalin }\end{array}$ \\
$\begin{array}{l}\text { Large aspiny } \\
\text { neurons }\end{array}$ & ACh \\
$\begin{array}{l}\text { INTERNEURONS } \\
\text { Cholinergic neurons }\end{array}$ & \\
GABAergic neurons & & \\
Somatostatin neurons & \\
\hline
\end{tabular}

Table 2: Discharge Properties of Single Neurons in the Basal Ganglia

Spontaneous activity

Putamen - silent at rest or low spontaneous firing rates

Globus pallidus - high frequency spontaneous discharges

Relation to voluntary movements

$50 \%$ of neurons discharge in association with limb movements

Close relationship to direction of movement

Variable temporal relationship to onset of movement

Some cells correlate with "motor set"

Responses to peripheral stimuli

Many cells show no response to any sensory input

Some cells respond to passive limb movement

No driving by cutaneous input

Microstimulation (of putamen)

Discrete movement of individual body parts on contralateral side 
unlikely that neurons in the putamen are involved in actually initiating movement. However, there is a subset of neurons in the putamen which is related in a complex way to the behavioural preparation for movement or what has been termed "motor set" ${ }^{6}$ These cells show changes in their firing patterns which

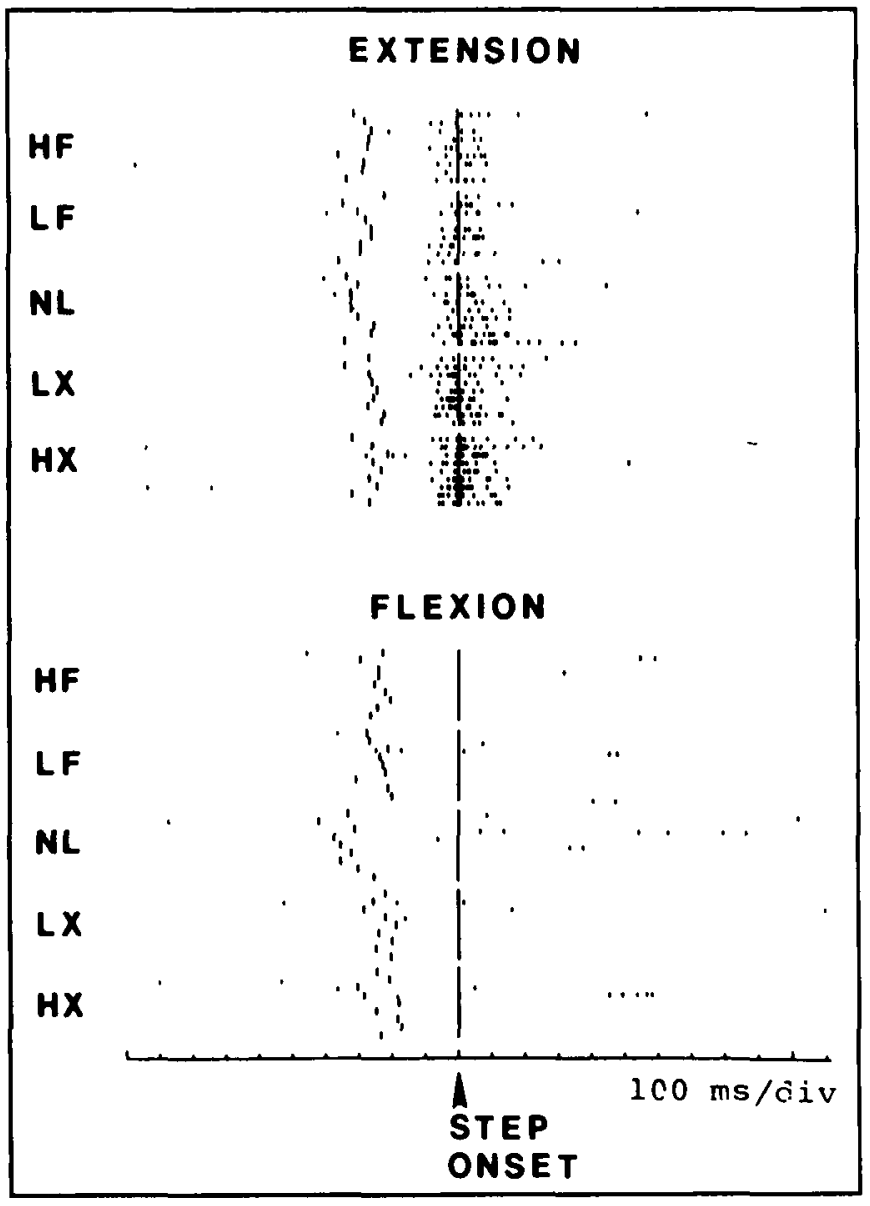

Figure 2 - Activity of a single neuron in the putamen of a monkey performing flexion-extension movements of the elbow with the contralateral arm. Each row of dots shows the timing of the spike discharges relative to movement onset for a single trial. The letters on the left indicate the amount and type of load which opposed the movement ( $H F$ and LF - heavy and light loads opposing flexion, $N L$ - No load, $L X$ and $H X$ - loads opposing extension (Reproduced from Crutcher and DeLong ${ }^{1.3}$ with permission).

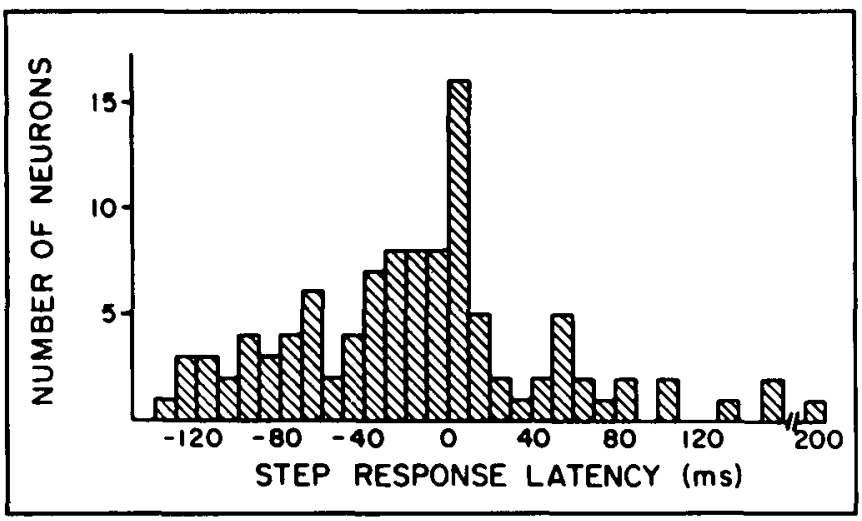

Figure 3 - Histogram to show times at which movement associated neurons in the monkey putamen change their firing frequency in relation to onset of movement which occurs at time zero (From Crutcher and DeLong ${ }^{13}$ with permission). precede movements in certain behavioural situations but not in others. For example, in a monkey which is trained to make a movement for which it receives a juice reward there are neurons in the putamen which increase their firing rate following the auditory signal which tells the animal to initiate the movement. If the reward is then not provided, within a few trials, the same auditory stimulus and the same movement is not accompanied by any changes in firing frequency of the cell. ${ }^{6}$

\section{Somatotopic organization}

Reconstruction of anatomical data to show the exact site of neurons which have been studied has confirmed that a precise somatotopic organization is maintained at all levels as information passes through the basal ganglia. "In the GPi and SNpr, which seem to be anatomically and physiologically one structure separated by part of the internal capsule, the leg is represented in the outer part of the GPi, the arm in the inner part, and the head and neck in SNpr. In the putamen cells are organised into functional clusters of $2-5$ neurons which show similar relationships to active or passive movement at a single joint. ${ }^{12}$ These clusters may correspond to the anatomically defined patches in putamen which have been demonstrated by labelling the terminals of projecting pathways.

As already mentioned the basal ganglia receive no direct sensory inputs from the periphery. Many cells which show changes in firing patterns associated with voluntary movement do not respond to any type of sensory input. Those which do tend to respond to passive joint movement and other forms of "deep" stimulation. In contrast to the cerebellum, neurons in the basal ganglia cannot be driven by cutaneous inputs.

\section{Microstimulation studies}

Standard electrical stimulation at various sites in the basal ganglia has produced conflicting and confusing results. Recently, however, Alexander and DeLong ${ }^{14,15}$ have demonstrated that microstimulation within the putamen produces discrete movements involving individual body parts, usually on the contralateral side. An example of their results is shown in Figure 4. This shows movement amplitude and velocity as well as associated EMG activity from the brachialis muscle in a monkey in which elbow flexion movements were produced in response to microstimulation of the contralateral putamen. Alexander and DeLong found that movements could be evoked by microstimulation at approximately $20 \%$ of sites studied in the putamen. No movement occurred in response to stimulation at any site in the caudate, reinforcing the concept that the caudate does not play a role in motor function and may be concerned with more complex behavioural activity .

\section{Pathophysiology of Parkinson's Disease}

Another approach which has been taken to help understand the function of the basal ganglia is physiological analysis of the clinical disorders which occur as a result of disease or injury affecting this area of the brain. Basal ganglia disorders can be considered in two major categories: those characterised by poverty of movement or akinesia, the obvious example being Parkinson's disease, and those characterised by excessive involuntary movements, the best example here being chorea. The most extensive studies have been done on Parkinson's disease. ${ }^{4,16}$ Some of these results will be reviewed briefly here. 


\section{Reaction times}

Simple reaction times to both visual and kinesthetic stimuli are prolonged in parkinsonian patients. ${ }^{17}$ However, parkinsonians do not show any additional impairment in choice reaction time experiments where they are required to respond to two signals, the second one specifying the direction of movement. Parkinsonian patients therefore seem capable of assembling motor programs in response to sensory stimuli. There is evidence that they may have difficulty modifying or replacing these programs if the initial response to the stimulus is not the correct one. This was demonstrated in an experiment by Angel ${ }^{18}$ who found a marked delay in changing direction when parkinsonian patients initiated a movement in the wrong direction in response to a false cue.

\section{Performance on tracking tasks}

Parkinsonian patients are more dependent on visual feedback than normal subjects when they carry out certain types of movement. ${ }^{19}$ This might account for some of the problems which parkinsonians have in performing arm movements to track a visual target moving in a sinusoidal pattern. Flowers has interpreted this defect as an inability for parkinsonians to adopt a predictive mode for tracking. ${ }^{20}$ According to Flowers parkinsonian patients "seem to lack a dynamic internal model of their own movements from which to control them predictably". However, in a subsequent set of experiments Day and Marsden ${ }^{21}$ concluded that "failure of predictive motor action is not an inevitable feature of Parkinson's disease".

\section{Ballistic movements}

With akinesia being one of the major clinical manifestations of parkinsonism, we might expect the most prominent abnormalities to show up on very fast or so called ballistic voluntary movements. This has been demonstrated in a number of experiments. The inability to generate high velocity movements is particularly obvious when parkinsonian patients attempt to perform large amplitude movements. Frequently they stop short of the target and then have to generate an additional movement.$^{20}$ Part of the reason for this is apparent when one examines the EMG activity associated with this type of movement. Hallett and Khosbin ${ }^{22}$ found that parkinsonian patients have difficulty generating a sufficiently large initial agonist EMG burst to reach a target with a single movement. Additional EMG bursts are required and the movements may end up consisting of a series of small steps. It has been proposed that this may be the basic mechanism underlying the bradykinesia of parkinsonism. ${ }^{22}$

Figure 5 shows an example of this type of abnormality in parkinsonian patient attempting to perform rapid wrist flexion movements to a specified target. Examination of the position and velocity records show that most of the movements are relatively slow despite the fact that the subject was instructed to move as rapidly as possible. In several trials the movement stops or even reverses direction before reaching the target zone. In the EMG recordings the initial agonist burst in the wrist flexors is not well defined and often consists of two or more segments. There is some tonic EMG activity in the extensors in all trials, but a well defined antagonist burst is seen only rarely. Parkinsonian patients seem to have difficulty recruiting a sufficient number of synchronized motor units to generate rapid large amplitude movements. It is also apparent in Figure 5 that there is marked variability in the pattern of EMG activation and the associated movement properties in successive single trials. Normal subjects show a fairly stereotyped EMG pattern from one trial to the next in a motor task of this type.

\section{Performance of simultaneous movements}

Additional abnormalities are apparent in parkinsonism if subjects are required to carry out more complex movements. It has been recognised for a long time that parkinsonian patients have difficulty performing more than one motor task at the same time. This phenomenon was re-examined recently by Benecke, et al. ${ }^{23}$ who had subjects perform two simple tasks - a rapid flexion of the elbow and an isometric squeeze of a force transducer held between the thumb and index finger. In normal subjects the speed of the individual movements was much the same whether they were performed independently or simultaneously. As expected parkinsonian patients were slower than normal subjects at performing either task independently but the time required to complete a movement was markedly increased when the patient attempted to perform the two tasks simultaneously. Benecke, et al. interpret these results as suggesting that in Parkinson's disease there may be a deficit in superimposing two separate motor programs.

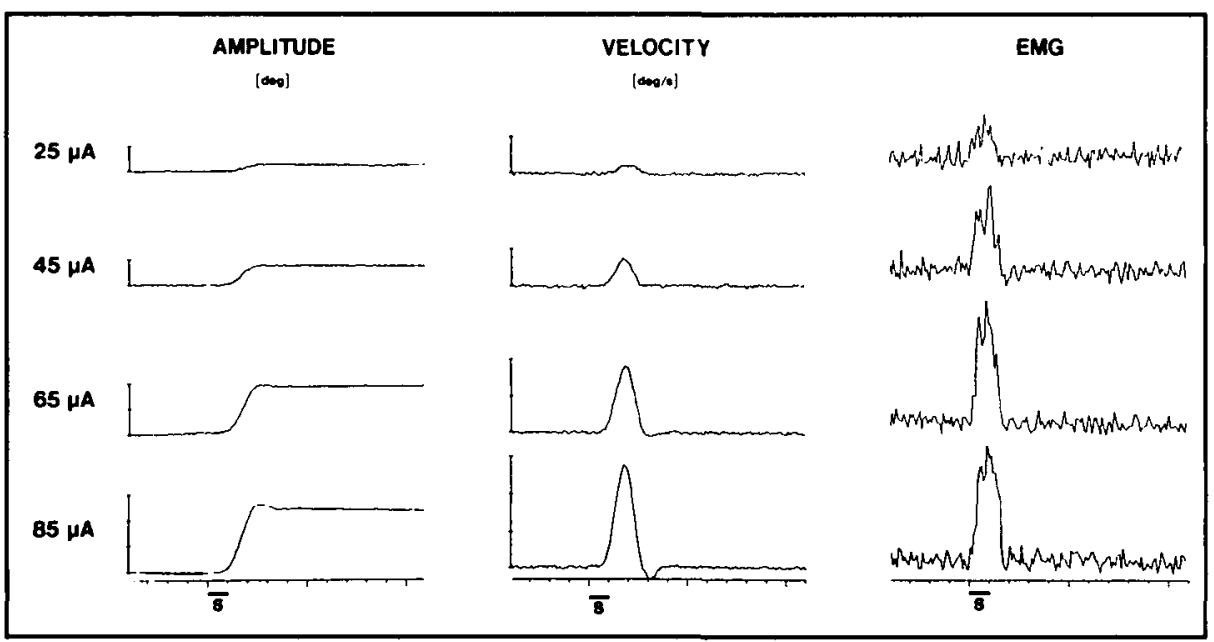

Figure 4 - Movement characteristics and EMG activity from the brachialis muscle associated with elbow flexions produced by microstimulation of the contralateral putamen at different stimulus strengths (From Alexander and DeLong ${ }^{14}$ with permission). 


\section{Long latency reflexes}

A consistent finding in many parkinsonian patients has been accentuation of the long latency component (M2) of the stretch reflex. ${ }^{24-25}$ In addition, the ability to modulate this late component according to prior instruction or volitional set seems to be lost in parkinsonian patients. Although there has been much debate concerning the origin of the $\mathrm{M} 2$ reflex component, there is strong evidence to support the view that it is mediated at least in part by a long loop transcortical pathway, and it was proposed that defective gain control in this feedback loop might be one of the mechanisms responsible for parkinsonian rigidity. ${ }^{24,26}$ Subsequent observations seemed to confirm that the $\mathrm{M} 2$ response was large in parkinsonian patients with prominent rigidity but not in those in whom rigidity was not a major feature. ${ }^{27}$ Other investigators however, have questioned whether there is any consistent correlation between the amount of rigidity and the size of M2. ${ }^{28}$ An interesting sequel to this story has been the observation by Noth $^{29}$ that $\mathrm{M} 2$ is reduced in patients with Huntington's disease, a condition in which there is also suppression of somatosensory evoked potentials.

\section{What Do the Basal Ganglia Do?}

Despite the rapid accumulation of knowledge concerning the basic structure and cellular physiology of the basal ganglia, any attempt to answer this question still requires considerable speculation. The basal ganglia obviously do many different things and it would be an oversimplification to attempt to assign any single function to such a complex area. These concluding comments will summarize some of the major concepts which have been discussed concerning the motor function of the basal ganglia.
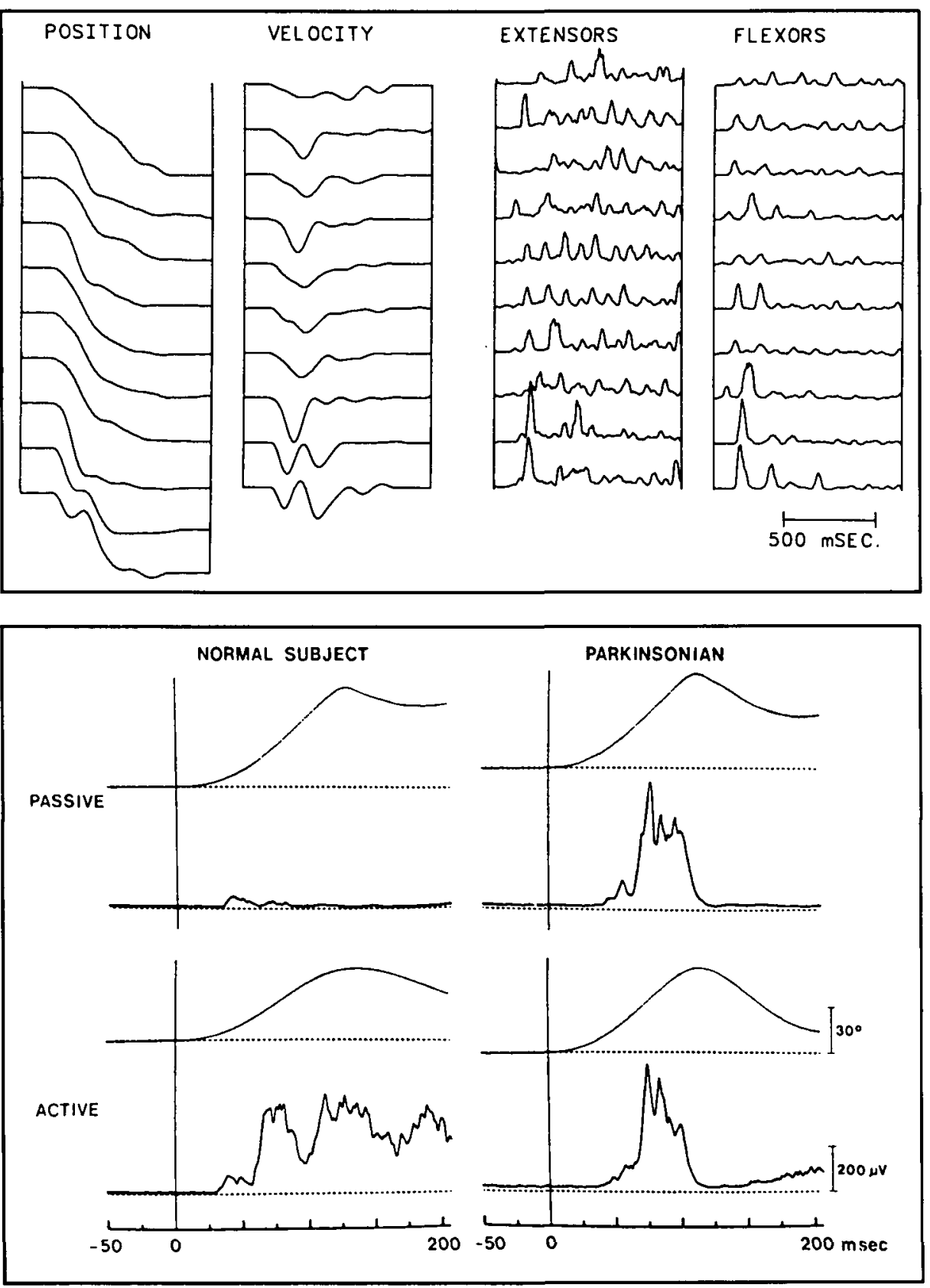

Figure 5-Recordings from a parkinsonian patient performing a series of wrist flexion movements to a target located $30^{\circ}$ from the starting position. The subject was attempting to move as rapidly as possible. EMG activity was recorded by surface electrodes over the wrist flexor and wrist extensor muscles.
Figure 6-Averaged EMG responses from the wrist flexors of a normal subject and a parkinsonian patient occcurring in response to extension perturbations at the wrist. The upper traces of each pair show movement at the wrist joint. The upper set of recordings for each subject show responses to passive extension of the wrist; the lower recording show responses when the subject actively opposes the displacement. Note the large $M 2$ component of the EMG response in the parkinsonian patient beginning about $60 \mathrm{msec}$ after the onset of perturbation (From Lee and Tatton ${ }^{25}$ with permission). 
Marsden ${ }^{4}$ has proposed that the basal ganglia are responsible for "the automatic execution of the sequence of motor programs which comprise a learned motor plan". This concept, which is based to a large extent on observations of the motor deficits in patients with Parkinson's disease, implies that complex motor activity or learned motor plans require the assembly of a number of different motor programs or sub-routines. There is no evidence that these programs are stored in the basal ganglia, but Marsden suggests that the basal ganglia may be responsible for collecting programs from other centres, assembling them into the proper sequence and then transmitting the package to cerebral cortex to allow the motor plan to be executed in an automatic manner. This is an interesting unifying hypothesis of basal ganglia motor function, but it may need to be broken down into smaller components before it can be adequately tested.

From their extensive studies on discharge properties of single neurons DeLong and his colleagues have concluded that the basal ganglia are responsible for specifying the direction and scaling the amplitude of voluntary movement. ${ }^{7,13}$ This proposed function would fit fairly well with some of the movement abnormalities observed in Parkinson's disease, particularly the difficulty performing large amplitude ballistic movements. However, it seems unlikely that this is an exclusive function of the basal ganglia, since neurons at several other sites in the central nervous system show similar correlations with movement direction and amplitude.

A different approach has been taken by Penney and Young ${ }^{3}$ who have proposed that the basal ganglia are involved in "selection of which behaviours will be carried out and suppression of unwanted ones". This proposal provides a convenient explanation for some of the involuntary movements seen with disorders of the basal ganglia. For example, chorea could be interpreted as failure to suppress unwanted movements. However, the specific mechanisms by which appropriate behaviours are selected and unwanted movements are suppressed are not at all clear at this stage from any of the experimental studies on basal ganglia function.

Finally, we shall return to the suggestion by Evarts and Wise ${ }^{6}$ that the basal ganglia "provide a pathway whereby cortical regions involved in certain higher brain functions gain access to motor control mechanisms". Since so much of human behaviour is expressed by movement, it would seem logical that there should be some mechanism for connecting centres concerned with mood, motivation, and memory to motor output areas. Whether the basal ganglia actually form this connecting link is still not fully established, but with the increased attention being focused on the basal ganglia by both basic and clinical neuroscientists, answers to these questions should be available in the fairly near future.

\section{REFERENCES}

1. DeLong MR, Georgopoulos AP. Motor functions of the basal ganglia. In: Brooks VB, ed. Handbook of Physiology, section I, Vol 2. Motor Control. Amer Physiol Soc 1981; 1017-1061.

2. Evarts EV, O'Connor M, eds. Function of the basal ganglia, (Ciba Foundation Symposium 107) Pitman, London 1984.
3. Penney JB Jr, Young AB. Speculations on the functional anatomy of basal ganglia disorders. Ann Rev Neurosci 1983; 6: 73-94.

4. Marsden CD. The mysterious motor function of the basal ganglia: the Robert Wartenberg Lecture. Neurology 1982; 32: 514-539.

5. Lee RG. Physiology of the basal ganglia: an overview. Can J Neurol Sci 1984; 11: 124-128.

6. Evarts EV, Wise SP. Basal ganglia outputs and motor control. In: Functions of the Basal Ganglia, (Ciba Foundation Symposium 107) Pitman, London 1984; 83-102.

7. Alexander GE, DeLong MR, Strick PL. Parallel organisation of functionally segregated circuits linking basal ganglia and cortex. Ann Rev Neurosci 1986; 9: 357-381.

8. Tracey DJ, Assanuma C, Jones EC, Porter R. Thalamic relays to motor cortex: afferent pathways from brainstem cerebellum and spinal cord in monkeys. J Neurophysiol 1980; 44: 532-554.

9. Bolam JP. Synapsis of identified neurons in the neostriatum. $\ln$ : Functions of the Basal Ganglia, (Ciba Foundation Symposium 107) Pitman, London $1984 ; 30-47$.

10. Nieoullon A, Kerkerian L, Dusticier N. Presynaptic controls in the neostriatum: reciprocal interactions between the nigro-strial dopaminergic neurons and the cortico-striatal glutamatergic pathway. In: Massion J, et al., eds. Neural Coding of Motor Performance. Springer-Verlag, Berlin 1983; 54-65.

11. DeLong MR, Georgopoulos AP. Motor function of the basal ganglia as revealed by studies of single cell activity in the behaving primate. Adv Neurol 1979; 24: 131-140.

12. Crutcher MD, DeLong MR. Single cell studies of the primate putamen. I. Functional organisation. Exp Brain Res 1984; 53: 233-243.

13. Crutcher MD and DeLong MR. Single cell studies of the primate putamen. II. Relations to direction of movement and pattern of muscular activity. Exp Brain Res 1984; 53: 244-258.

14. Alexander GE and DeLong MR. Microstimulation of the primate neostriatum. I. Physiological properties of striatal microexcitable zones. J Neurophysiol 1985; 53: 1417-1432.

15. Alexander GE, DeLong MR. Microstimulation of the primate neostriatum II Somatotopic organization of striatal microexcitable zones and their relation to neuronal response properties. J Neurophysiol 1985; 53: 1433-1436.

16. Marsden $C D$. Which motor disorder in Parkinson's disease indicates the true motor function of the basal ganglia? In: Functions of the Basal Ganglia, (Ciba Foundation Symposium 107) Pitman, London 1984 ; 225-241.

17. Evarts EV, Teravainen H, Calne DB. Reaction time in Parkinson's disease. Brain 1981; 104: 167-186.

18. Angel AW, Alston W, Higgins JR. Control of movement in Parkinson's disease. Brain 1970; 93: 1-14.

19. Cooke JD, Brown JD. Increased dependence on visual information for arm movement in patients with Parkinson's disease. In: Poirier LJ, Sourkes TL, Bedard PJ, eds. The Extrapyramidal System and its Disorders, Raven Press, New York 1979; 185-189.

20. Flowers KA. Visual "closed-loop" and "open-loop" characteristics of voluntary movement in patients with parkinsonian and intention tremor. Brain 1976; 99: 269-310.

21. Day BL, Marsden CD. Two strategies for learning a visually guided motor task. Percept Motor Skills 1982; 55: 1003-1016.

22. Hallett M, Khosbin S. A physiological mechanism of bradykinesia. Brain 1980; 103: 301-314.

23. Benecke R, Rothwell JC, Dick JPR, et al. Performance of simultaneous movements in patients with Parkinson's disease. Brain 1986; 109: 739-757.

24. Lee RG, Tatton, WG. Motor responses to sudden limb displacement in primates with specific CNS lesions and in human patients with motor system disorders. Can J Neurol Sci 1975; 2: 285-293.

25. Lee RG, Tatton WG. Long loop reflexes in man: clinical applications. In: Desmedt JE, ed. Progress in Clinical Neurophysiology, Cerebral Motor Control in Man, Long Loop Mechanisms Karger, Basel 1978; 4: 320-333.

26. Lee RG, Murphy JT, Tatton WG. Long latency myotatic reflexes in man: mechanisms, functional significance, and changes in patients with Parkinson's disease or hemiplegia. In: Desmedt JE, ed. Motor Control Mechanisms in Health and Disease. Adv Neurol 1983; 39: 489-508. 
27. Tatton, WG, Beddingham W, Verrier MC, Blair RDJ. Characteristic alterations in responses to imposed wrist displacements in parkinsonian rigidity and dystonia musculorum deformans. Can J Neurol Sci 1984; 11: 281-287.

28. Cody FWJ, MacDermott N, Matthews PBC, Richardson HC. Obser- vations on the genesis of the stretch reflex in Parkinson's disease. Brain 1986; 109: 229-249.

29. Noth J, Podoll K, Friedmann H-H. Long-loop reflexes in small hand muscles studied in normal subjects and in patients with Huntington's disease. Brain 1985; 108: 65-80. 